\title{
A Novel Robust Mesh Watermarking Based on BNBW
}

\author{
Liping Chen,, ${ }^{1,2}$ Xiangzeng Kong, ${ }^{1}$ Bin Weng, ${ }^{1}$ Zhiqiang Yao, ${ }^{1,3}$ and Rijing Pan ${ }^{1}$ \\ ${ }^{1}$ Key Laboratory of Network Security and Cryptology, Fujian Normal University, Fuzhou 350007, China \\ ${ }^{2}$ College of Mathematics and Computer Science, Fujian Normal University, Fuzhou, Fujian 350007, China \\ ${ }^{3}$ Faculty of Software, Fujian Normal University, Fuzhou 350007, China \\ Correspondence should be addressed to Xiangzeng Kong, xzkongfjnu@sohu.com
}

Received 15 June 2010; Revised 27 October 2010; Accepted 15 February 2011

Academic Editor: Dimitrios Tzovaras

Copyright ( 2011 Liping Chen et al. This is an open access article distributed under the Creative Commons Attribution License, which permits unrestricted use, distribution, and reproduction in any medium, provided the original work is properly cited.

\begin{abstract}
As a solution to copyright protection of the digital media, digital watermarking techniques have been developed for embedding specific information to identify the owner in the host data imperceptibly. Nowadays, most watermarking methods mainly focused on digital media such as images, video, audio, and text, and very few watermarking methods have been presented for 3D models relatively. In the paper, a new robust watermarking scheme is presented which is based on biorthogonal nonuniform B-spline wavelets (BNBW) in the frequency domain for the purpose of copyright protection in the area of CAD, CAM, CAE, and CG. The watermark is embedded by modulating the wavelet coefficient vectors with the watermark in the frequency domain. The relative experiments prove that this approach not only can withstand common attacks on 3D models such as polygon mesh simplifications, addition of random noise, model cropping, translation, rotation, scaling, as well as a combination of such attacks but also can detect and locate tampered vertices.
\end{abstract}

\section{Introduction}

The digital media have been widely used to create many digital products. For example, people can obtain, duplicate, process, and distribute the digital media relatively easily by many of the existing tools and the Internet. As a result, these facilities are also exploited by pirates who use them illegally for their personal gains to violate the legal rights of the digital content providers. The digital watermarking has been introduced as an effective complementary to the traditional encryption for the digital watermark could be embedded into the various kinds of digital media, including images, audio data, video data, and three-dimensional graphical models such as 3D polygonal models. Most of the previous researches have focused on general types of multimedia data, including text data, images, audio data, and video data until 1997, when Ohbuchi proposed 3D mesh model watermarking algorithm $[1,2]$ for first time. Recently, with the interest and requirement of 3D models such as VRML (virtual reality modeling language) data, CAD (computer aided design) data, polygonal mesh models, and medical objects, 3D model watermarking has received much attention in the community, and considerable progress has been made. Several watermarking techniques for 3D models have been introduced [3-8].

Theoretically, watermarking algorithms can fall into two categories: spatial-domain methods and frequency-domain methods. In the spatial-domain methods, the watermark is embedded directly by modifying the positions of vertices, the colors of texture points or other elements representing the model. While in the frequency-domain methods the watermark is embedded by modifying the transform coefficients. For the spatial-domain algorithms the researchers embed watermarking into certain 3D model invariants like triangle similarity quadruple (TSQ), tetrahedral volume ratio (TVR) $[1,2,9,10]$, affine invariant embedding (AIE) [11, 12], and so forth. But most of these algorithm are very sensitive to noise. Most of frequency-domain algorithms provide better robustness. They use wavelet analysis $[13,14]$ and Laplace transforms $[15,16]$ to embed watermarking. They can embed one bit watermarking into the whole 3D model.

Kanai et al. [13], in 1998, proposed the first mesh watermarking scheme based on wavelet analysis. The scheme decomposed a $3 \mathrm{D}$ polygon mesh into a multiresolution representation by performing lazy wavelet transform proposed by Lounsbery et al. [17]. Uccheddu et al. [14] extend 
[13] by detecting the watermark without the original mesh. Both of them cannot process irregular meshes directly because of the limitation of Lounsbery's scheme [17]. Some other trials using multiresolution scheme also have been introduced in paper [18-21]. The multiresolution techniques could achieve good transparency of watermark except for the solution for various synchronization attacks such as vertex reordering, remeshing, and simplification. Now, we propose a new robust watermarking scheme based on the biorthogonal nonuniform B-spline wavelets (BNBW) transform. The novelty of this paper lies in that the scheme not only can be applied to both regular and irregular 3D model but also can be against the various attacks including the synchronizational attacks and topological attacks. The proposed scheme can embed the watermark even into the irregular ones, which overcomes the drawback of only embedding the watermark into regular meshes in the article $[13,14,17]$. Furthermore, the scheme extends the various normal attacks in which the algorithm [18-21] can resist to the synchronizational attacks.

The rest of this paper is organized as follows. In Section 2, we introduce some related works including wavelet analysis for 3D meshes and conventional wavelet analysisbased watermarking methods. In Section 3, we explain the watermark insertion and extraction algorithms in detail. In Section 4, we show some of our experimental results. Finally, in Section 5, we conclude and mention potential improvement in future work.

\section{Related Works}

2.1. Wavelet Analysis. Wavelet analysis is one of the most useful multiresolution representation techniques which are used in a broad range of applications such as image compression, physical simulation, and numerical analysis. Kanai et al. [13] extended wavelet analysis to mesh watermarking scheme in 1998. The wavelet analysis scheme simplifies the original meshes by reversing a subdivision scheme. The simplification is repeated as hard as possible. The original mesh $\mathbf{V}_{0}$ is decomposed into the multiresolution representation by applying the wavelet transform at several times. In the multiresolution representation, $V_{0}$ is decomposed both into the set of wavelet coefficient vectors $W_{1}, W_{2}, \ldots, W_{d}$ at every resolution level, and into the coarsest approximation $V_{d}$, where $d$ means the coarsest resolution level. Typically, we simplify the mesh to a suitable coarsest resolution level, and then the watermark information is embedded into wavelet coefficient vectors or the coarsest approximation. Finally, we can get the watermarked mesh $\hat{\mathbf{V}}_{\mathbf{0}}$ by inverse wavelet transform.

2.2. Biorthogonal Nonuniform B-Spline Wavelets. The biorthogonal nonuniform B-spline wavelets is a kind of multiresolution representation scheme proposed by Pan and Yao [22], and the article $[23,24]$ also is about B-spline wavelets of multiresolution representation. We briefly introduce the Biorthogonal nonuniform B-spline wavelets for meshes in the following; more detailed descriptions can be found in [22] or in the Appendix of this paper.

We consider nonuniform B-spline wavelets of order $k$ on finite interval $[a, b]$. Let $\mathbf{T}_{0} \subset \mathbf{T}_{1} \subset \cdots$ be a nested sequence of knot vectors, where $\mathbf{T}_{i}=\left\{t_{i, 0}, t_{i, 1}, \ldots, t_{i, n_{i}+k}\right\}, i=0,1, \ldots$ satisfy the following conditions:

$$
\begin{aligned}
a & =t_{i, 0}=\cdots=t_{i, k-1}<t_{i, k} \leq t_{i, k+1} \leq \cdots \leq t_{i, n_{i}}<t_{i, n_{i}+1} \\
& =\cdots=t_{i, n_{i}+k}=b, t_{i, j}<t_{i, j+k}, \quad j=0,1, \ldots, n_{i}, n_{i} \geq k-1 .
\end{aligned}
$$

Suppose that $\left\{N_{i, j, k}(t)\right\}_{j=0}^{n_{i}}$ is the normalized B-spline basis of order $k$ determined by knot vector $\mathbf{T}_{\mathbf{i}}$. Then, $\mathbf{V}_{\mathbf{i}}=$ $\operatorname{Span}\left\{N_{i, 0, k}(t), N_{i, 1, k}(t), \ldots, N_{i, n_{i}, k}(k)\right\}, i=0,1, \ldots$ constitute a nested sequence of polynomial spline spaces of degree $k-1$, that is, $\mathbf{V}_{0} \subset \mathbf{V}_{1} \subset \cdots$. On the basis of it, a MRA of the Bspline wavelets can be established.

Let $\mathbf{W}_{i}$ be a complement space of $\mathbf{V}_{i}$ in $\mathbf{V}_{i+1}$; that is, $\mathbf{V}_{i+1}=\mathbf{V}_{i}+\mathbf{W}_{i}$ and $\left\{\Psi_{i, j}(t)\right\}_{j=1}^{m_{i}}$ be a basis of $\mathbf{W}_{i}$, where $m_{i}+n_{i}=n_{i+1}$. Then, $\left\{\Psi_{i, j}(t)\right\}_{j=1}^{m_{i}}$ is a set of the nonuniform B-spline wavelets. Let

$$
\mathbf{N}_{i, k}=\left\lfloor N_{i, 0, k} N_{i, 1, k} \cdots N_{i, n_{i}, k}\right\rfloor
$$

and let $\Psi_{i}=\left[\Psi_{i, 1} \Psi_{i, 2} \cdots \Psi_{i, m_{i}}\right]$. Then, there exist matrices $\mathbf{P}_{i}$ of order $\left(n_{i+1}+1\right) \times\left(n_{i}+1\right)$ and $\mathbf{Q}_{i}$ of order $\left(n_{i+1}+1\right) \times m_{i}$ such that

$$
\left\lfloor\mathbf{N}_{\mathbf{i}, \mathbf{k}} \quad \Psi_{i}\right\rfloor=\mathbf{N}_{i+1, k}\left[\begin{array}{ll}
\mathbf{P}_{i} & \mathbf{Q}_{i}
\end{array}\right]
$$

where $\mathbf{P}_{i}$ and $\mathbf{Q}_{i}$ are called the reconstruction matrices of the B-spline wavelets. Let $\left[\begin{array}{l}\mathbf{A}_{i} \\ \mathbf{B}_{i}\end{array}\right]=\left[\begin{array}{ll}\mathbf{P}_{i} & \mathbf{Q}_{i}\end{array}\right]^{-1}$.

Then, we have $\mathbf{N}_{i+1, k}=\left[\begin{array}{ll}\mathbf{N}_{i, k} & \Psi_{i}\end{array}\right]\left[\begin{array}{l}\mathbf{A}_{i} \\ \mathbf{B}_{i}\end{array}\right]$, where matrices $\mathbf{A}_{i}$ of order $\left(n_{i}+1\right) \times\left(n_{i+1}+1\right)$ and $\mathbf{B}_{i}$ of order $m_{i} \times\left(n_{i+1}+\right.$ 1) are called the decomposition matrices of the B-spline wavelets.

For any $f_{i+1}=\mathbf{N}_{i+1, k} \mathbf{d}_{i+1} \in \mathbf{V}_{i+1}, f_{i+1}$ can be uniquely decomposed into the lower resolution part $\mathbf{f}_{i}=\mathbf{N}_{i, k} \mathbf{d}_{i} \in \mathbf{V}_{i}$ and the detail part $g_{i}=\Psi_{i} \mathbf{w}_{i} \in \mathbf{W}_{i}$ by decomposition matrices $\mathbf{A}_{i}$ and $\mathbf{B}_{i}$; that is, $f_{i+1}=f_{i}+g_{i}$, where

$$
\mathbf{d}_{i}=\mathbf{A}_{i} \mathbf{d}_{i+1}, \quad \mathbf{w}_{i}=\mathbf{B}_{i} \mathbf{d}_{i+1} .
$$

On the other hand, using $\mathbf{P}_{i}$ and $\mathbf{Q}_{i}, f_{i+1}$ can be reconstructed by $f_{i}$ and $g_{i}$

$$
\mathbf{d}_{i+1}=\mathbf{P}_{i} \mathbf{d}_{i}+\mathbf{Q}_{i} \mathbf{w}_{i}
$$

Hence, the key of the MRA based on B-spline wavelets is the construction of reconstruction matrices $\mathbf{P}_{i}$ and $\mathbf{Q}_{i}$ as well as decomposition matrices $\mathbf{A}_{i}$ and $\mathbf{B}_{i}$. The computation of $\mathbf{A}_{i}$ and $\mathbf{B}_{i}$ is dependent on reconstruction matrices $\mathbf{P}_{i}$ and 
$\mathbf{Q}_{i}$. For all kinds of B-spline wavelets, $\mathbf{P}_{i}$ 's all knot insertion matrices. They can be computed by Olso Algorithm or recursive algorithm, and so forth. But for different B-spline wavelets, $\mathbf{Q}_{i}$ is different. So, the challenge is to construct $\mathbf{Q}_{i}$ for the construction of B-spline wavelets.

Since semiorthogonal wavelets require that wavelet space $\mathbf{W}_{i}$ is orthogonal to scale space $\mathbf{V}_{i}$, and the orthogonality is defined by the inner product $\langle f, \mathrm{~g}\rangle=\int_{a}^{b} f(t) g(t) d t$ of space $L_{2}[a, b]$, a large amount of integral calculations are involved in the computation of $\mathbf{Q}_{i}$. In order to avoid integral operation, we abandon the orthogonality defined by continuous norm $L_{2}$. Alternately, we construct biorthogonal wavelets. An essential point is to define orthogonality of $\mathbf{W}_{i}$ and $\mathbf{V}_{i}$ by discrete norm $l_{2}$ for vectors, that is, to define discrete inner product of space $\mathbf{V}_{i}$ as $\left\langle f_{i}, h_{i}\right\rangle=\mathbf{d}_{i}^{T} \mathbf{s}_{i}$, where $f_{i}=\mathbf{N}_{i, k} \mathbf{d}_{i}, h_{i}=\mathbf{N}_{i, k} \mathbf{s}_{i}$. Then, from (3), we know the conditions that reconstruction matrix $\mathbf{Q}_{i}$ should satisfy are column full rank and the following discrete orthogonal condition:

$$
\mathbf{P}_{i}^{T} \mathbf{Q}_{i}=\mathbf{0},
$$

where $\mathbf{0}$ is the Zero-matrix of order $\left(n_{i}+1\right) \times m_{i}$. The method for the construction of $\mathbf{Q}_{i}$ is given in Section 4 .

According to (4)-(6), the lower resolution coefficient vector $\mathbf{d}_{i}$ and the wavelet coefficient vector $\mathbf{w}_{i}$ are the least square solutions of (7) and (8), respectively,

$$
\begin{aligned}
\mathbf{P}_{i} \mathbf{x} & =\mathbf{d}_{i+1}, \\
\mathbf{Q}_{i} \mathbf{x} & =\mathbf{d}_{i+1} .
\end{aligned}
$$

Then, according to (4), the decomposition matrices are given as following:

$$
\begin{aligned}
\mathbf{A}_{i} & =\mathbf{P}_{\mathbf{i}}^{+}=\left(\mathbf{P}_{\mathbf{i}}^{T} \mathbf{P}_{i}\right)^{-1} \mathbf{P}_{i}^{T}, \\
\mathbf{B}_{i} & =\mathbf{Q}_{\mathbf{i}}^{+}=\left(\mathbf{Q}_{\mathbf{i}}^{T} \mathbf{Q}_{i}\right)^{-1} \mathbf{Q}_{i}^{T},
\end{aligned}
$$

where $\mathbf{P}_{i}^{+}$and $\mathbf{Q}_{i}^{+}$are the generalized inverse matrices of $\mathbf{P}_{i}$ and $\mathbf{Q}_{i}$, respectively, satisfying $\mathbf{P}_{\mathbf{i}}^{+} \mathbf{P}_{\mathbf{i}}=\mathbf{I}_{\left(n_{i}+1\right) \times\left(n_{i}+1\right)}$ and $\mathbf{Q}_{\mathbf{i}}^{+} \mathbf{Q}_{\mathbf{i}}=\mathbf{I}_{m_{i} \times m_{i}}$.

Thus, (3), (6), and (10) are the all conditions that reconstruction matrices and decomposition matrices should satisfy for the proposed biorthogonal nonuniform B-spline wavelets.

\section{The Principium of the Scheme}

3.1. Watermark Embedding Process. The basic procedures of watermarking scheme are shown in Figure 1. The steps of the watermark embedding process are as follows. (a) Convert Cartesian coordinates of a vertex $v_{i}=$ $\left(x_{i}, y_{i}, z_{i}\right)$ of original mesh model $V$ into spherical coordinates $\left(\rho_{i}, \theta_{i}, \phi_{i}\right)$ by

$$
\begin{gathered}
\rho_{i}=\sqrt{\left(x_{i}-x_{g}\right)^{2}+\left(y_{i}-y_{g}\right)^{2}+\left(z_{i}-z_{g}\right)^{2}}, \\
\theta_{i}=\tan ^{-1} \frac{\left(y_{i}-y_{g}\right)}{\left(x_{i}-x_{g}\right)}, \\
\phi_{i}=\cos ^{-1} \frac{\left(z_{i}-z_{g}\right)}{\sqrt{\left(x_{i}-x_{g}\right)^{2}+\left(y_{i}-y_{g}\right)^{2}+\left(z_{i}-z_{g}\right)^{2}}},
\end{gathered}
$$

where $0 \leq i \leq N-1, N$ is the number of the vertex, and $\left(x_{g}, y_{g}, z_{g}\right)$ is the center of gravity of the mesh model. The proposed scheme uses only vertex norms $\rho_{i}$ for watermarking and keeps the other two components $\theta_{i}$ and $\phi_{i}$ intact. The distribution of vertex norms is obviously invariant to vertex reordering and similarity transforms.

(b) The vertices are divided into $S$ distinct sections by $\theta_{i}$ and $\phi_{i}$ with the same range. Each section must be suitable to embed all watermarks independently. As a result the watermark can be embedded repeatedly $S$ times into different sections.

(c) For each section, the norms $\rho_{i}$ are arranged ascendingly as $R\left(\rho_{0}, \rho_{1} \cdots \rho_{L-1}\right)$, where $L$ is the number of the vertex. And then, the $\mathrm{B}$-spline knot vectors $\mathbf{T}_{0}=$ $\left\{t_{0,0}, t_{0,1}, \ldots, t_{0, n_{i}+k}\right\}$ are computed with $R\left(\rho_{0}, \rho_{1} \cdots \rho_{L-1}\right)$ by Hartley-Judd algorithm. Then, Biorthogonal nonuniform B-spline wavelets (see Section 2.2) analysis is performed forward with the $\mathrm{B}$-spline knot vectors $\mathrm{T}$. In this way, a set of the wavelet coefficient vector $W_{k}\left(\bar{\rho}_{0}, \bar{\rho}_{1} \cdots \bar{\rho}_{m_{k}-1}\right)$ are obtained at approximation (resolution) level $k$ which can be determined by considering the capacity and the invisibility of the watermark embedding.

(d) Embedded the watermark into wavelet coefficient vector $W_{k}\left(\bar{\rho}_{0}, \bar{\rho}_{1} \cdots \bar{\rho}_{m_{k}-1}\right)$ by modifying the wavelet coefficient as follows:

$$
\tilde{\rho}_{i}=\bar{\rho}_{i}+\alpha \bar{\rho}_{i} w_{i} \quad 0 \leq i \leq m-1
$$

The watermark $w_{i} \in\{-1,1\}$, whose length is $m$, is embedded into $\bar{\rho}_{i}$ proportion to $\bar{\rho}_{i}$ with the global strength factor $\alpha$, which can help to extract the watermark easily, but it has to be selected properly, because it also controls the visual quality after embedding the watermark.

(e) Execute the inverse Biorthogonal nonuniform Bspline wavelets transform. Meanwhile, the B-spline knotvectors $\widetilde{\mathbf{T}}$ can be computed with reconstruction matrices $\mathbf{P}$ and $\mathrm{Q}$ by the method proposed in Section 2.2. Moreover, the new $\widetilde{R}\left(\tilde{\rho}_{0}, \tilde{\rho}_{1} \cdots \tilde{\rho}_{L-1}\right)$ are contructed to get the new vertex spherical coordinates $\widetilde{v}_{i}=\left(\tilde{\rho}_{i}, \theta_{i}, \phi_{i}\right)$. 


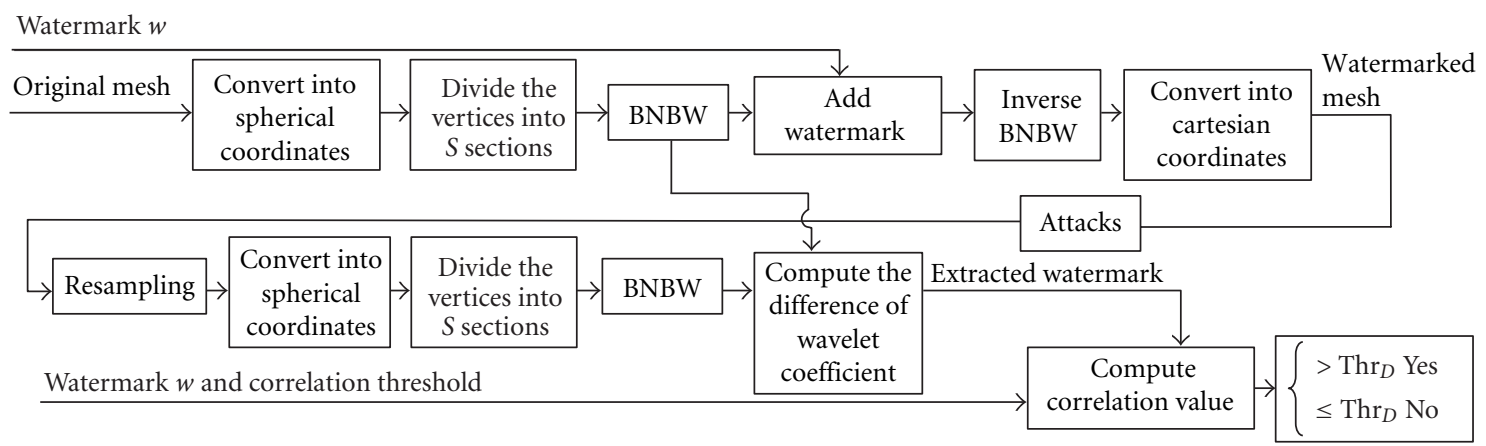

FIgURE 1: Outline of the proposed BNBW-based watermarking method.

(f) Convert the spherical coordinates to Cartesian coordinates. The Cartesian coordinates $\left(\tilde{x}_{i}, \tilde{y}_{i}, \tilde{z}_{i}\right)$ of vertex $\tilde{v}_{i}$ on stego mesh model is given by

$$
\begin{gathered}
\tilde{x}_{i}=\tilde{\rho}_{i} \cos \theta_{i} \sin \phi_{i}+x_{g}, \\
\tilde{y}_{i}=\tilde{\rho}_{i} \sin \theta_{i} \sin \phi_{i}+y_{g}, \\
\tilde{z}_{i}=\tilde{\rho}_{i} \cos \phi_{i}+z_{g},
\end{gathered}
$$

where $0 \leq i \leq L-1, \theta_{i}, \phi_{i}$ and the center of gravity are the same as those calculated in the step (a). Finally, the watermarked mesh model $V^{\prime}$ can be obtained.

3.2. Watermark Extracting Process. Figure 1 is the outline of the proposed BNBW-based watermarking method. The steps of the watermark extracting process are as follows.

(a) The detected model resampling: the resampling procedure is as follows: in the beginning, a ray is cast from the center of the original model to the original vertex $V_{o i}$ and intersect with the detected model. If the ray intersects the watermarked model at one or more points and point $V_{d i}$ is the closest intersection point to $V_{o i}$, then $V_{d i}$ is taken as the vertex that corresponds with $V_{o i}$, or let $V_{d i}=V_{o i}$.

(b) As in steps (a) of the embedding procedure, Cartesian coordinates of a vertex $v_{i}^{\prime}=\left(x_{i}, y_{i}, z_{i}\right)$ of original mesh model $V^{\prime}$ are converted into spherical coordinates $\left(\rho_{i}^{\prime}, \theta_{i}^{\prime}, \phi_{i}^{\prime}\right)$.

(c) As in steps (b) of the embedding procedure, the vertices are divided into $S$ distinct sections by $\theta_{i}^{\prime}$ and $\phi_{i}^{\prime}$ with equal range.

(d) As in steps (c) of the embedding procedure, the biorthogonal nonuniform B-spline wavelets analysis is performed to obtain a set of the wavelet coefficient vector $W_{k}\left(\rho_{0}^{\prime}, \rho_{1}^{\prime} \cdots \rho_{m_{k}-1}^{\prime}\right)$ at corresponding (resolution) level $k$.

(e) Perform forward biorthogonal nonuniform B-spline wavelets analysis with original mesh $V$ as the steps of the embedding procedure, so that the wavelet coefficient vector $W_{k}\left(\bar{\rho}_{0}, \bar{\rho}_{1} \cdots \bar{\rho}_{m_{k}-1}\right)$ at level $k$ can be got. Furthermore, compute the difference between wavelet coefficient of the watermarked mesh model
$V^{\prime}$ and wavelet coefficient of original mesh model $V$ as follows:

$$
D_{i j}=\rho_{i j}^{\prime}-\bar{\rho}_{i j}
$$

where $\bar{\rho}_{i j}$ is the $i$ th BNBW wavelet coefficient of $j$ th sections of original mesh model and $\rho_{i j}^{\prime}$ is the $i$ th BNBW wavelet coefficient of $j$ th sections of watermarked mesh model. $D_{i j}$ is the difference betwee $\rho_{i j}^{\prime}$ and $\bar{\rho}_{i j}$.

(f) Extract watermark. The watermark has been embedded repeatedly $S$ times into different sections in the process of embedding. So, we decide the watermark as follows:

$D_{i}=\sum_{j=0}^{S-1} D_{i j}, \quad w_{i}^{\prime}=\operatorname{sign}\left(D_{i}\right) \quad 0 \leq i \leq m-1$.

The sign is a function that returns the sign of its parameter.

(g) Compute the correlation between the extracted watermark sequence and the designated watermark sequence to decide whether the designated watermark is presented in the detected model

$$
\begin{aligned}
& \operatorname{Cor}\left(W^{\prime}, W\right) \\
& =\frac{\sum_{i=0}^{M-1}\left(w_{i}^{\prime}-\bar{W}^{\prime}\right)\left(w_{i}-\bar{W}\right)}{\sqrt{\sum_{i=0}^{M-1}\left(w_{i}^{\prime}-\bar{W}^{\prime}\right)^{2}+\sum_{i=0}^{M-1}\left(w_{i}-\bar{W}\right)^{2}}},
\end{aligned}
$$

where $W^{\prime}$ is the extracted watermark sequence, $W$ is the designated watermark sequence, $\bar{W}^{\prime}$ is the mean value of $W^{\prime}, \bar{W}$ is the mean value of $W$, and $M$ is the length of the watermark sequence. If the computed correlation value exceeds a chosen threshold $\mathrm{ThrD}$, we conclude that the designated watermark is present in the detected model.

\section{Experimental Results}

In order to test our watermarking technique, we conduct experiments on a triangle of a Venus model. The Venus 


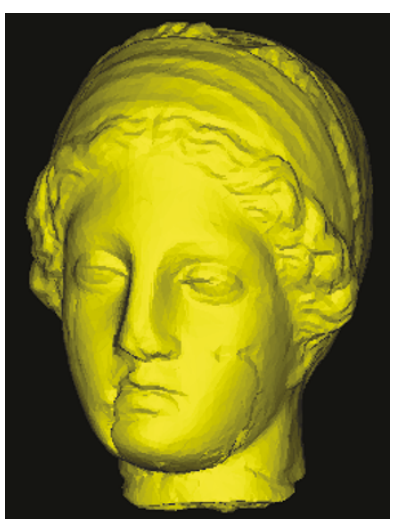

(a)

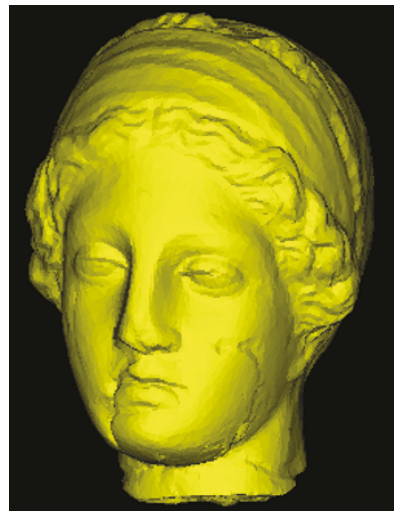

(b)

Figure 2: (a) Original model. (b) Watermarked model.
TABLE 1: Results of simplification attacks.

\begin{tabular}{lccc}
\hline Removing ratio & $30 \%$ & $50 \%$ & $70 \%$ \\
\hline Venus cor & 1.0 & 0.8451 & 0.5342 \\
Horse cor & 1.0 & 0.7975 & 0.4750 \\
Bunny cor & 1.0 & 0.8738 & 0.6459 \\
\hline
\end{tabular}

TABLE 2: Results of cropping and noise attacks.

\begin{tabular}{lccc}
\hline Cropping ratio & $30 \%$ & $50 \%$ & $70 \%$ \\
\hline Venus cor & 0.9128 & 0.8743 & 0.6029 \\
Horse cor & 0.8751 & 0.7951 & 0.5752 \\
Bunny cor & 0.9017 & 0.8871 & 0.5147 \\
\hline
\end{tabular}

model consists of 10002 vertices and 20000 triangle faces. The length of the original watermarking sequence $N$ is 40 , and we set the Parameter $S=50$. So, The bit capacity that was tested is $40 * 30=1200$. The PSNR (peak signal to noise ratio) between the original and the watermarked mesh model and BER (bit error rate) of detected watermark information are adopted to test the imperceptibility and the robustness, respectively. The PSNR is defined as

$$
D_{i j}=\rho_{i j}^{\prime}-\bar{\rho}_{i j} .
$$

The watermarked Venus model is shown in Figure 2(b), and the Figure 2(a) is the original Venus model. Visually comparing these two figures, we can conclude that the embedded watermark is imperceptible. Our proposed method is based on the wavelet transform and multiresolution representation of the 3D mesh model. The watermark can be embedded in the wavelet coefficient vectors at the various resolution levels of the multiresolution representation, which makes the embedded watermark imperceptible. The experiments are carried out both on the horse model and bunny model. We subject the watermarked Venus model to polygon simplification, noise, cropping operations, as well as combined attacks so as to test the robustness of our algorithm. The experimental results show that the algorithm is very robust against these attacks and can detect the integrality of the 3D model as detailed in the following.

To demonstrate our watermarking algorithm's resistance to noise, in our experiment, the noise is added to the watermarked model by perturbing its vertices at full resolution in a random way. Especially, different displacement vector $\Delta_{\text {noise }}=\left(\Delta_{x}, \Delta_{y}, \Delta_{z}\right)$ is applied for each vertex. The vector components $\Delta x, \Delta y$ and $\Delta_{z}$ are random variables with uniform distribution in the interval $[-\Delta, \Delta]$. In Figure 3 , $\Delta_{\text {noise }}$ is $0.3 \%, 0.6 \%$, and $1.2 \%$, respectively, of the distance of the longest vector extended from a vertex to the center of the model. In Figure 4, the value of $\rho$ and ThrD for increasing values of $\Delta_{\text {noise }}$ is given. Aiming to set an appropriate threshold value, we generate 1000 random watermark sequences whose length is 100 and then select 500 sequences randomly as the watermark to be embedded in to the $3 \mathrm{D}$ mesh model. Moreover, we calculate the linear correlation coefficient between the randomly generated watermarks and the original watermark. While the experiment indicates that the correlation values between the randomly generated watermarks and the original watermark are less than 0.45 , so the threshold $T$ was set to 0.5 . In particular, the plot is given as a function of the quantity $\Delta_{\text {noise }}$. The models used in this test are Venus watermarked at level of resolution $l=3$ with $\alpha=0.03$. The experimental results in Figure 4 show that the algorithm can resist these noise attacks very well.

For simplification attack, we simplify the watermarked bunny model with triangular faces. We reduce $30 \%, 50 \%$, and $70 \%$, of the triangular faces of the bunny model, respectively. We also carry out experiments on the horse model and Venus model. The experimental result is shown in Table 1 and Figure 5.

The robustness of the algorithm against the cropping attacks is tested in three different cases, which included removing $30 \%, 50 \%$, and $70 \%$ of the vertices in the watermarked bunny model, respectively. And $0.3 \%$ noise is add to some vertices of the vertices left. Because in each section we embedded a watermark bit has $S$ vertices, which means the watermarking scheme embed a watermark bit in different vertex for $S$ times, the result is the watermarking scheme can resist the crop attacks. The experiments are also carried out on the horse model and Venus head model, which are shown in Table 2 and Figure 6. These results again demonstrate that the algorithm is also robust against cropping attacks with high correlation values for the watermark extraction.

Furthermore, we have tested the algorithm's robustness against the geometry attack of translation, rotation, and scaling. Experimental results demonstrated that the algorithm is also robust against attack of translation, rotation, and scaling. And the proposed scheme uses only vertex norms $\rho_{i}$ for watermarking and keeps the other two components $\theta_{i}$ and $\phi_{i}$ intact. The distribution of vertex norms is obviously invariant to vertex reordering and similarity transforms. 


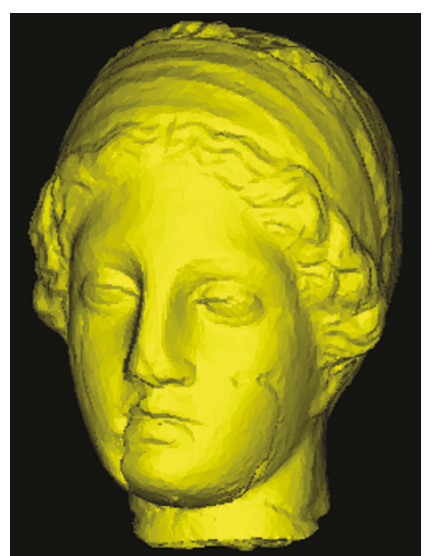

(a) $0.3 \%$

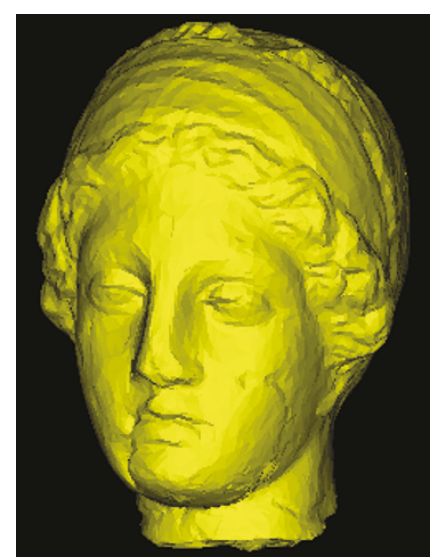

(b) $0.6 \%$

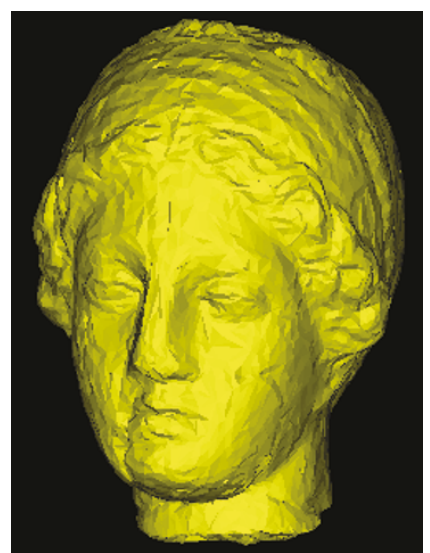

(c) $1.2 \%$

Figure 3: $(\mathrm{a}-\mathrm{c})$ add noise.

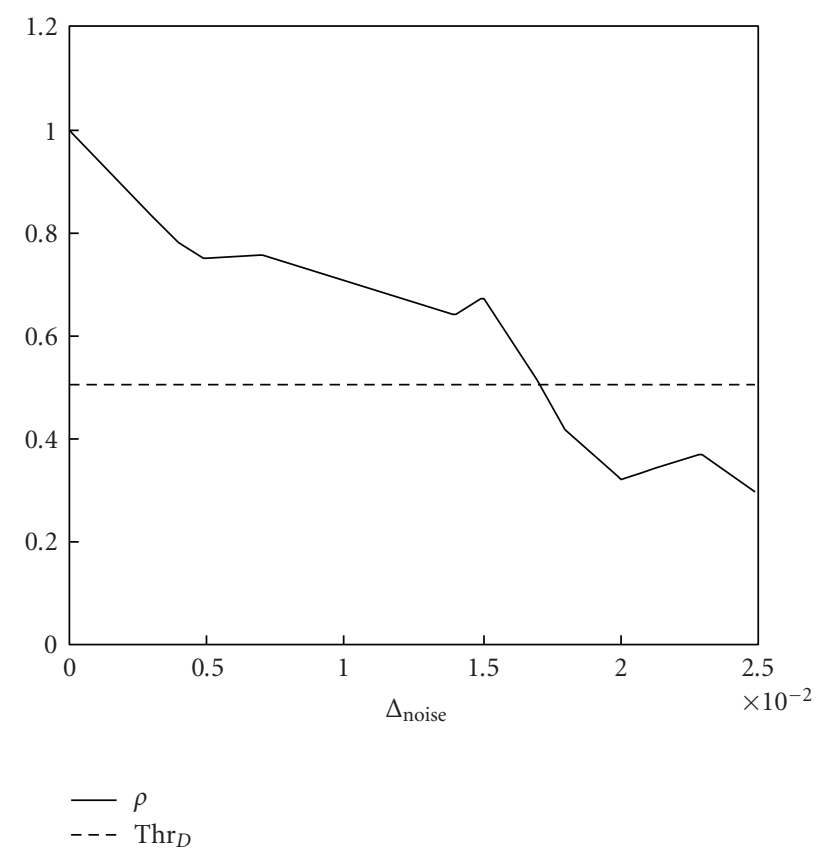

Figure 4: Robustness against additive noise attack.

\section{Conclusion and Future Work}

In the paper, a new robust watermarking scheme based on biorthogonal nonuniform B-spline wavelets (BNBW) in the frequency domain is presented for the purpose of copyright protection in the area of CAD, CAM, CAE, and CG. The watermark is embedded by modulating the wavelet coefficient vectors with the watermark in the frequency domain. In order to cast the watermarking problem in a multiresolution framework, the algorithm is extended to work with irregular meshes, thus making $3 \mathrm{D}$ wavelet analysis feasible. Experiments show that this approach not only is able to withstand common attacks on $3 \mathrm{D}$ models such as polygon mesh simplifications, addition of random noise, model cropping, translation, rotation, scaling, as well as a combination of such attacks but also can detect and locate tampered vertices.

Watermarking of 3D meshes has received a limited attention due to the difficulties encountered in extending the algorithms developed for 1D (audio) and 2D (images and video) signals to the topological complex objects such as meshes. Other difficulties lie in the wide variety of attacks and the robustness against the manipulations of 3D watermarks. For this reason, most of the 3D watermarking algorithms proposed adopted a nonblind detection, which is known as less useful in practical applications compared with the blind ones. In the future work, we intend to improve our algorithm to nonblind watermarking by embedding the side 


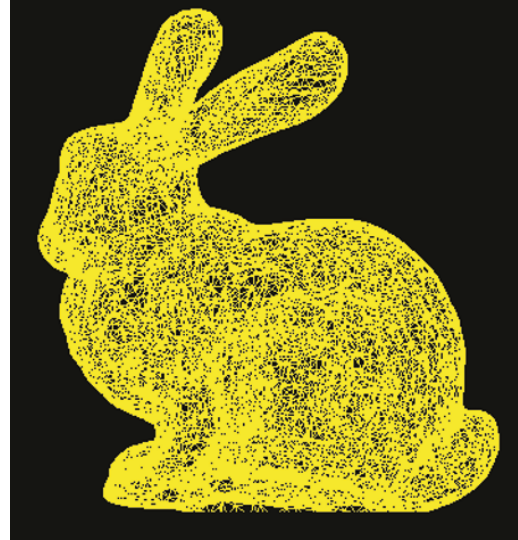

(a)

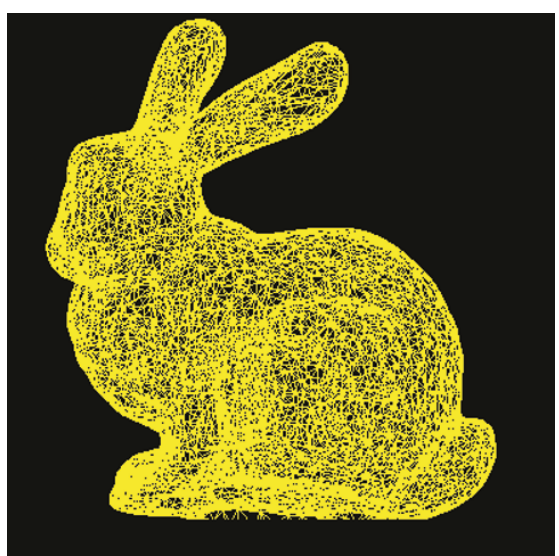

(b)

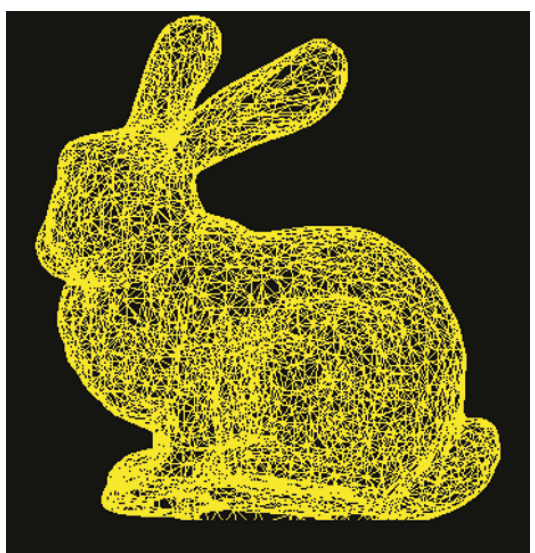

(c)

Figure 5: (a) 30\% (b) 50\% (c) 70\% triangular faces removed (simplified) from the watermarked 3D model.

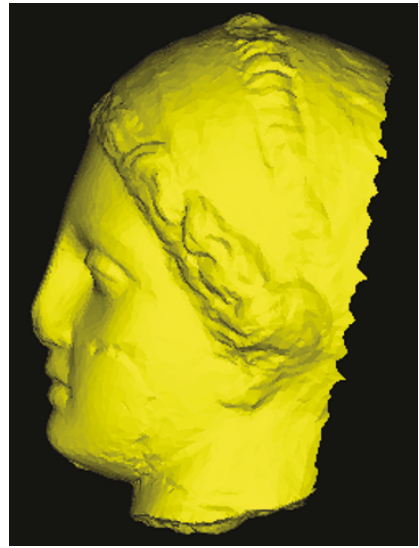

(a)

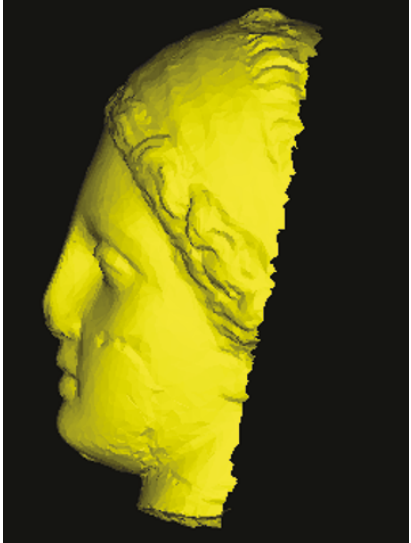

(b)

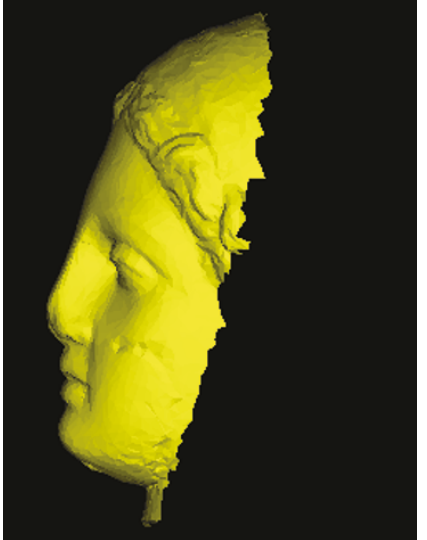

(c)

Figure 6: (a) 30\% (b) 50\% (c) 70\% faces cropped from the watermarked 3D model and 0.3\% noise.

information of original model information as the watermark of the model.

Several directions for future work remain open. First of all, we can apply other kinds of attacks and test possible failures of our algorithms. We can extend our method to undergoing general affine transformations although it can only undergoing similarity transformations at present. Secondly, we can upgrade our watermarking algorithm into a blind watermarking algorithm. Finally, the possibility of modulating the watermark strength according to perceptual considerations will be investigated so as to increase the imerceptuality of the watermark.

\section{Appendix}

\section{Reconstruction and Decomposition Algorithms}

Most of the content of this Appendix is derived from [22], in which Pan and Yao propose biorthogonal nonuniform B- spline wavelets based on a discrete norm. We hope this will facilitate the understandings of our method.

(1) Algorithm Reconstruction. The following is the reconstruction algorithm for biorthogonal nonuniform B-spline wavelets based on discrete norm $l_{2}$.

Input: order of $\mathrm{B}$-spline $k$, level no. $i$, lower resolution coefficient vector $\mathbf{d}_{i}$, wavelet coefficient vector $\mathbf{w}_{i}$, and knot vectors $\mathbf{T}_{i}$ and $\mathbf{T}_{i+1}$.

Output: reconstruction matrices $\mathbf{P}_{\mathbf{i}}$ and $\mathbf{Q}_{i}$, higher resolution coefficient vector $\mathbf{d}_{i+1}$.

(i) Let $\mathbf{T}=\mathbf{T}_{\mathbf{i}}, \overline{\mathbf{T}}=\mathbf{T}_{i+1}, n=n_{i}$, and $\bar{n}=n_{i+1}$.

(ii) Compute $\mathbf{P}_{\mathbf{i}}$ by equation as follows: 


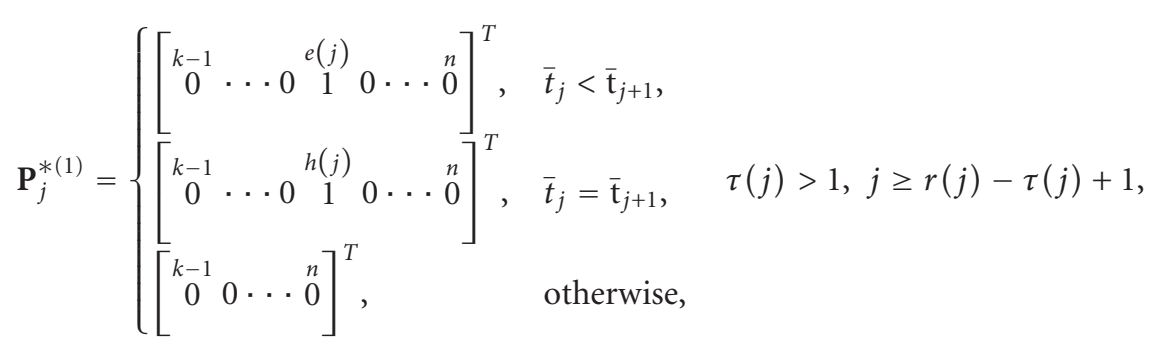

$j=k-1, \quad k, \ldots, \bar{n}$,

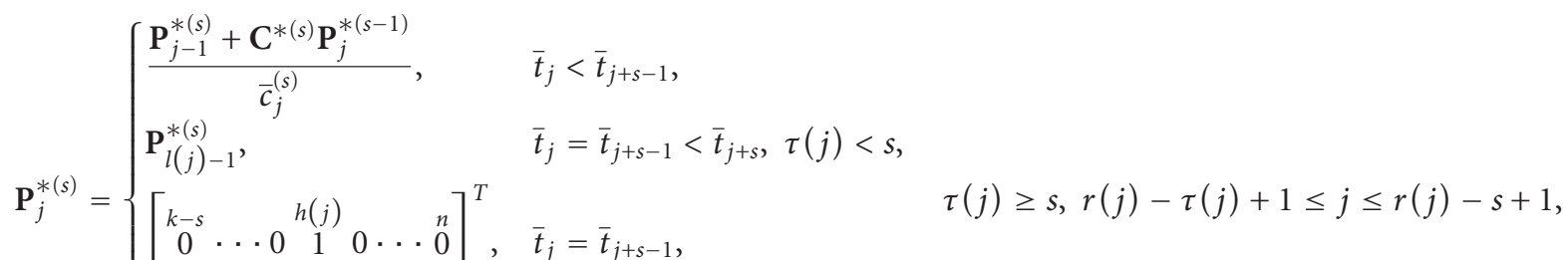

$$
\begin{aligned}
& {\left[\begin{array}{cccc}
k-1 & n & n \\
0 & 0 & \cdots & 0
\end{array}\right]^{T}, \quad \text { otherwise, }} \\
& j=k-s, k-s+1, \ldots,+\bar{n}, \quad s=2,3, \ldots, k .
\end{aligned}
$$

(iii) Compute $\mathbf{Q}_{i}$ by equation as follows:

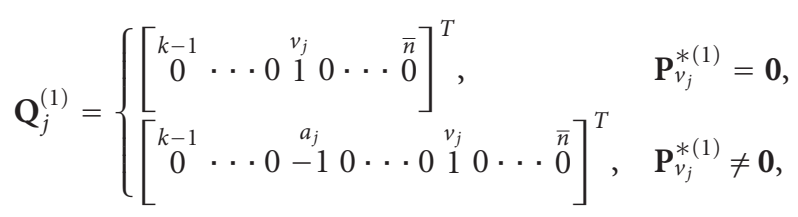

$$
\begin{aligned}
& j=1,2, \ldots, \bar{n}-n, \\
& \mathbf{Q}^{(s)}=\overline{\mathbf{C}}^{*(s)} \mathbf{Q}^{(s-1)}, \quad s=2,3, \ldots, k .
\end{aligned}
$$

(iv) Compute $\mathbf{d}_{i+1}=\mathbf{P}_{i} \mathbf{d}_{i}+\mathbf{Q}_{i} \mathbf{w}_{i}$.

(2) Algorithm Decomposition. The following is the decomposition algorithm for biorthogonal nonuniform B-spline wavelets based on discrete norm $l_{2}$.

Input: order of $\mathrm{B}$-spline $k$, level no. $i$, higher resolution coefficient vector $\mathbf{d}_{i+1}$, and reconstruction matrices $\mathbf{P}_{\mathbf{i}}$ and $\mathbf{Q}_{i}$.

Output: lower resolution coefficient vector $\mathbf{d}_{i}$ and wavelet coefficient vector $\mathbf{w}_{i}$.

(i) Solve linear equation system $\mathbf{P}_{i}^{\mathrm{T}} \mathbf{P}_{i} \mathbf{x}=\mathbf{P}_{i}^{T} \mathbf{d}_{i+1}$ by Gaussian elimination to obtain $\mathbf{d}_{i}$.

(ii) Solve linear equation system $\mathbf{Q}_{i}^{T} \mathbf{Q}_{i} \mathbf{x}=\mathbf{Q}_{i}^{T} \mathbf{d}_{i+1}$ by Gaussian elimination to obtain $\mathbf{w}_{i}$.
According to $\mathbf{d}_{i+1}=\mathbf{P}_{i} \mathbf{d}_{i}+\mathbf{Q}_{i} \mathbf{w}_{i}$, another method for decomposition is to solve the whole linear system

$$
\left[\begin{array}{ll}
\mathbf{P}_{i} & \mathbf{Q}_{i}
\end{array}\right]\left[\begin{array}{l}
\mathbf{d}_{i} \\
\mathbf{w}_{i}
\end{array}\right]=\mathbf{d}_{i+1} .
$$

The computation consists of two steps: firstly, a band coefficient matrix is obtained by exchanging its lows or columns, and then the system is solved with band structure.

\section{Acknowledgments}

This research work is supported by the National Natural Science Foundation of China under Grant no. 60673014 and NSF of Fujian under Grant no. 2008J0013. The authors would like to thank Dr. Pan and Dr. Yao for their valuable discussions and supports. They would also like to give our special thanks to the anonymous reviewers for their valuable comments and suggestions.

\section{References}

[1] R. Ohbuchi, H. Masuda, and M. Aono, "Embedding data in 3D models," in Proceedings of the European Workshop on Interactive Distributed Multimedia systems and Telecommunication Services, pp. 1-10, Darmstadt, Germany, 1997.

[2] R. Ohbuchi, H. Masuda, and M. Aono, "Watermarking threedimensional polygonal models," in Proceedings of the 5th ACM International Multimedia Conference, pp. 261-272, Seattle, Wash, USA, November 1997. 
[3] O. Benedens, "Geometry-based watermarking of 3D models," IEEE Computer Graphics and Applications, vol. 19, no. 1, pp. 46-55, 1999.

[4] C. M. Chou and D. C. Tseng, "A public fragile watermarking scheme for 3D model authentication," CAD Computer Aided Design, vol. 38, no. 11, pp. 1154-1165, 2006.

[5] M. Luo and A. G. Bors, "Principal component analysis of spectral coefficients for mesh watermarking," in Proceedings of the IEEE International Conference on Image Processing (ICIP '08), pp. 441-444, San Diego, Calif, USA, October 2008.

[6] J. W. Cho, R. Prost, and H. Y. Jung, "An oblivious watermarking for 3-D polygonal meshes using distribution of vertex norms," IEEE Transactions on Signal Processing, vol. 55, no. 1, pp. 142-155, 2007.

[7] M. Hu, Y. Xie, L. Xu, and F. Xue, "A geometry property based adaptive watermarking scheme for 3D models," Journal of Computer-Aided Design and Computer Graphics, vol. 20, no. 3, pp. 390-402, 2008.

[8] Y. Zhiqiang, Z. Rongchun, H. S. Ip Horace et al., "A robust watermarking scheme for 3D models," Computer Engineering and Applications, vol. 38, no. 2, pp. 23-27, 2002.

[9] R. Ohbuchi, H. Masuda, and M. Aono, "Watermarking three-dimensional polygonal models through geometric and topological modifications," IEEE Journal on Selected Areas in Communications, vol. 16, no. 4, pp. 551-559, 1998.

[10] R. Ohbuchi, H. Masuda, and M. Aono, "Data embedding algorithms for geometrical and non-geometrical targets in threedimensional polygonal models," Computer Communications, vol. 21, no. 15, pp. 1344-1354, 1998.

[11] O. Benedens and C. Busch, "Towards blind detection of robust watermarks in polygonal models," Computer Graphics Forum, vol. 19, no. 3, pp. C199-C208, 2000.

[12] O. Benedens, "Affine invariant watermarks for 3D polygonal and NURBS based models," in Proceedings of the 3rd International Workshop on Information Security (ISW'00), pp. 15-29, 2000.

[13] S. Kanai, H. Date, and T. Kishinami, "Digital watermarking for 3D polygons using multiresolution wavelet decomposition," in Proceedings of the 6th IFIP WG 5.2 GEO-6, pp. 296-307, Tokyo, Japan, 1998.

[14] F. Uccheddu, M. Corsini, and M. Barni, "Wavelet-based blind watermarking of 3D models," in Proceedings of the Multimedia and Security Workshop (MM\&Sec '04), pp. 143154, September 2004.

[15] R. Ohbuchi, A. Mukaiyama, and S. Takahashi, "A frequencydomain approach to watermarking 3D shapes," Computer Graphics Forum, vol. 21, no. 3, pp. 373-382, 2002.

[16] F. Cayre, P. Rondao-Alface, F. Schmitt, B. Macq, and H. Maître, "Application of spectral decomposition to compression and watermarking of 3D triangle mesh geometry," Signal Processing: Image Communication, vol. 18, no. 4, pp. 309-319, 2003.

[17] M. Lounsbery, T. D. DeRose, and J. Warren, "Multiresolution analysis for surfaces of arbitrary topological type," ACM Transactions on Graphics, vol. 16, pp. 34-73, 1997.

[18] K. Yin, Z. Pan, J. Shi, and D. Zhang, "Robust mesh watermarking based on multiresolution processing," Computers and Graphics, vol. 25, no. 3, pp. 409-420, 2001.

[19] J. Q. Jin, M. Y. Dai, H. J. Bao, and Q. S. Peng, "Watermarking on 3D mesh based on spherical wavelet transform," Journal of Zhejiang University, vol. 5, no. 3, pp. 251-258, 2004.

[20] M. S. Kim, S. Valette, HO. Y. Jung, and R. Prost, "Watermarking of $3 \mathrm{D}$ irregular meshes based on wavelet multiresolution analysis," in Proceedings of the International Workshop on
Digital Watermarking, vol. 3710 of Lecture Notes in Computer Science, pp. 313-324, 2005.

[21] M. S. Kim, J. W. Cho, R. Prost, and H. Y. Jung, "Wavelet analysis based blind watermarking for 3-D surface meshes," in Proceedings of the International Workshop on Digital Watermarking (IWDW'06), vol. 4283 of Lecture Notes in Computer Science, pp. 123-137, 2006.

[22] R. Pan and Z. Yao, "Biorthogonal nonuniform B-spline wavelets based on a discrete norm," Computer Aided Geometric Design, vol. 26, no. 4, pp. 480-492, 2009.

[23] D. Li, K. Qin, and H. Sun, "Curve modeling with constrained B-spline wavelets," Computer Aided Geometric Design, vol. 22, no. 1, pp. 45-56, 2005.

[24] G. Zhao, S. Xu, W. Li, and O. E. Teo, "Fast variational design of multiresolution curves and surfaces with B-spline wavelets," CAD Computer Aided Design, vol. 37, no. 1, pp. 73-82, 2005. 